\title{
HIGH-PERFORMANCE PROTEIN PRECONCENTRATOR USING MICROCHANNEL-INTEGRATED NAFION STRIP
}

\author{
H. Yang, M. Shen, V. Sivagnanam and M. A. M. Gijs \\ Laboratory of Microsystems, École Polytechnique Fédérale de Lausanne, Lausanne, SWITZERLAND
}

\begin{abstract}
A microfluidic device for protein preconcentration based on the electrokinetic trapping principle is proposed. It comprises a Nafion strip which is simply cut from a commercially available membrane and integrated into a polydimethylsiloxane (PDMS) microfluidic structure using a guiding channel. By applying low voltage $(<15$ $\mathrm{V})$, the device exhibits a preconcentration factor of $>10^{3}$ of fluorescently labeled bovine serum albumin (BSA) within a few minutes. Moreover, a numerical study of the preconcentration effect is presented to microscopically understand the phenomenon in our type of device.
\end{abstract}

\section{KEYWORDS}

Protein preconcentrator; electrokinetic trapping; Nafion; microfluidics.

\section{INTRODUCTION}

Microfluidic devices have shown to provide privileged solutions for many problems in chemical and biological analysis, especially for in-field use or point-of-care testing [1]. However, micro-scale analysis devices can only handle limited volumes, which pose limits to practical applications. One issue is that certain target biomolecules are present at very low concentration, so that the accurate determination of their presence or concentration is challenging. Therefore, efficient sample purification/preconcentration methods are necessary.

Among various techniques available for biomolecules preconcentration in microfluidic devices, electrokinetic trapping has been studied by many research groups [2-3]. It is an electric field-addressable trapping principle, and it can be used for any charged biomolecule and allows the use of simple fluid-handling protocols. However, electrokinetic trapping usually calls for specially fabricated nanoporous membranes [3-4] or nanofluidic channels [5-6]. Though nanofluidic channels have proved to efficiently concentrate biomolecules in different type of devices, cumbersome fabrication steps and extreme control of surface charge in the nanofluidic chip are necessary. Compared to this, a commercially available ion-exchange membrane (Nafion) can overcome complex fabrication process and assembly problems. Here we propose a rapid-prototyped, simple-structured microfluidic device based on electrokinetic trapping for protein preconcentration. We simply cut a Nafion strip from a commercially available membrane and integrate it into a PDMS channel [7]. Our device achieves a preconcentration factor of $10^{3}-10^{4}$ for various concentrations of BSA within $10 \mathrm{~min}$. For microscopic understanding of the working mechanism, we present a numerical simulation by simultaneously solving the Nernst-Planck, Poisson and Navier-Stokes equations for our device.

\section{DESIGN AND WORKING PRINCIPLE}

The proposed preconcentrator consists of a glass substrate that is mechanically clamped with a PDMS chip which has two microfluidic channels separated by a guiding channel for inserting the Nafion strip. The nanoporous Nafion strip, with fixed negative charges and mean pore size on the order of electric double layer (EDL), is utilized as a cation-permselective membrane. When placed between two electrodes in an electrolyte, cations can migrate through the membrane to their corresponding electrode, while anions cannot. Figure 1 demonstrates the working principle of the device schematically. The microfluidic channel is separated into the upper sample channel and the lower buffer channel by Nafion nanoporous membrane strip, forming the anodic and cathodic sides of the device, respectively. The fluidic reservoirs of the cathodic compartment are connected to a voltage $V_{B}=0 \mathrm{~V}$, while the reservoirs of the anodic compartment have two different positive voltages $V_{H}$ and $\mathrm{V}_{\mathrm{L}}$, respectively. While a bias voltage is applied, the electrical field across the Nafion membrane $\left(E_{n}\right)$ generates ion concentration polarization $(\mathrm{CP})$, with ion depletion at the anodic side and ion enrichment at the cathodic side. A local excess of mobile positive ions is generated in the depleted $\mathrm{CP}$ zone, which is very close to the membrane and induces a non-equilibrium electrical double layer (EDL). In this case, the electroosmotic flow (EOF) inside the upper channel consists of two parts, i.e. the EOF induced by the fixed charges on the channel walls (1st EOF), and the EOF induced by the space charge of the non-equilibrium EDL close to the membrane (2nd EOF). Meanwhile, the electrophoretic (EP) force generated by electrical field $\left(\mathrm{E}_{\mathrm{t}}\right)$ due to the voltage difference $\left(\mathrm{V}_{\mathrm{H}}-\mathrm{V}_{\mathrm{L}}\right)$ over the anodic channel acts on the BSA tracer molecules, which makes the latter move opposite to the EOF. Therefore, the co-effect of EOF and EP provides the basis for the enrichment of the tracer biomolecules.

\section{EXPERIMENTS}

\section{Microfluidic Device Fabrication}

The process flow of the microfluidic protein preconcentrator is shown in Figure 2. The PDMS chip is fabricated through a standard replica molding procedure with a master of $\sim 170 \mu \mathrm{m}$ thick negative photoresist SU-8 on silicon substrate. The Nafion is cut into a $200 \mu \mathrm{m}$ wide 


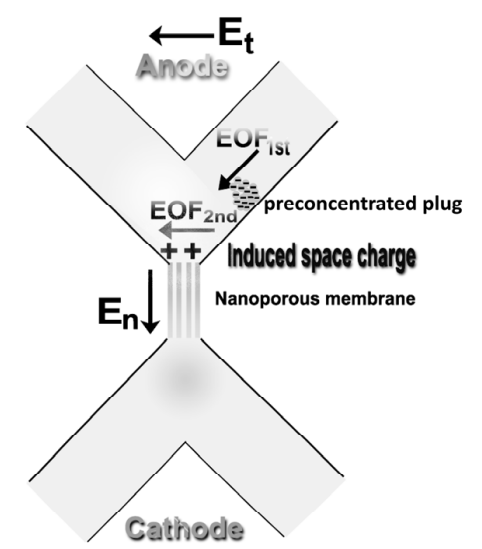

Figure 1: Schematic diagram of the physics of the concentration polarization under bias voltages.

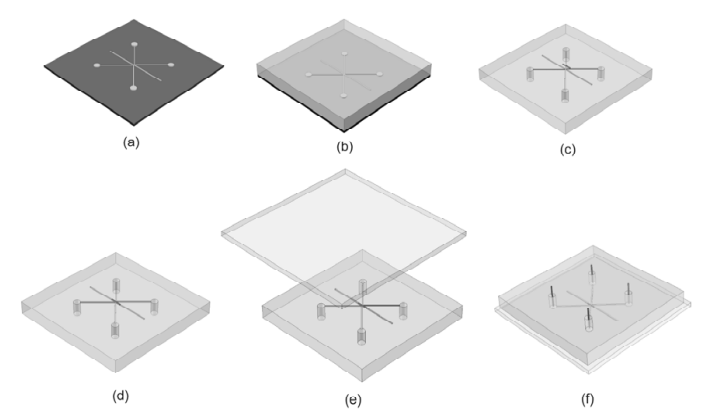

Figure 2. Fabrication flow for the Nafion strip-integrated preconcentrator: (a) SU-8 master, (b) liquid PDMS pouring and curing, (c) peeling off PDMS and cutting the Nafion strip, (d) Nafion strip integration, (e) placing the glass substrate, (f) chip assembly with inserted electrodes.

strip by using two razor blades, and subsequently a standard pretreatment procedure is used to prepare Na-form Nafion [8]. Afterwards, the pre-cleaned PDMS chip is lightly bended and the integration of the Nafion strip is realized thanks to the guiding channel. The assembly of the PDMS microstructure and glass substrate is achieved by a mechanical clamping technique using poly(methyl methacrylate) (PMMA) support sheets, enabling leakage-free functioning of the chip. Finally, four Pt wires are placed into the four fluidic reservoirs as electrodes.

\section{Experimental Materials and Protocols}

For preconditioning, the microfluidic channel is first washed by a PBS (phosphate buffered saline) -Tween (PBST) solution ( $\mathrm{pH}$ 7.4) using syringes. The PBST solution is prepared by mixing $0.5 \%(\mathrm{v} / \mathrm{v})$ Tween-20 (Sigma-Aldrich Chemie $\mathrm{GmbH}$, Buchs, Switzerland) with $1 \times$ PBS, which is diluted from $10 \times$ concentrated solution purchased from Sigma-Aldrich. Then $1 \times$ PBS is loaded into the buffer channel, while the sample channel is blocked with PBS-BSA $(1 \% \quad(\mathrm{w} / \mathrm{v}) \quad \mathrm{BSA}$ (Sigma-Aldrich) in $1 \times$ PBS) solution for $\sim 5 \mathrm{~min}$. This step is essential to avoid the unspecific adsorption of the fluorescently-labeled BSA molecules on the microchannel walls. Following this, the PBS-BSA solution is replaced by $1 \times$ PBS. Finally, BSA conjugated with Alexa Fluor 647 (AF-BSA, Invitrogen, Basel, Switzerland) in $1 \times$ PBS is filled into the sample channel for the preconcentration experiments.

During the experiment, the Pt electrodes in the buffer reservoirs are connected to zero potential, while a bias voltage, $\mathrm{V}_{\mathrm{H}}-\mathrm{V}_{\mathrm{L}}$, is applied across the sample channel through the Pt electrodes in the sample reservoirs. For fluorescence image acquisition, we use an ORCA-C4742-95ER CCD camera (Hamamatsu, Shizuoka Prefecture, Japan) mounted on an inverted microscope Axiovert S100 (Zeiss). The microscope is equipped with a mercury short arc lamp, HBO (Osram), and filter set for Alexa Fluor 647. The exposure time used for capturing the fluorescence signal is 2 seconds, and AquaCosmos software (Hamamatsu) is used for the fluorescence image analysis.

\section{Experimental Results}

In the preconcentration experiment, the most important parameters are the applied voltage across the membrane, the voltage difference $\left(\mathrm{V}_{\mathrm{H}}-\mathrm{V}_{\mathrm{L}}\right)$ across the sample channel, preconcentration factor, as well as the tracer protein concentrations. We will discuss these parameters specifically in the following paragraphs.

To clarify the influence of the potential difference $\left(\mathrm{V}_{\mathrm{H}}-\mathrm{V}_{\mathrm{L}}\right)$ and quantify the preconcentration factor, we measured the fluorescent intensity of the preconcentrated plug as a function of time for different potential differences at the same initial AF-BSA concentration, as shown in Figure 3. The data points and error bars correspond to average fluorescent intensity and variance, respectively. A sampling area of 16 pixels around the maximum is used to record the experimental data. From Figure 3, the higher potential difference $\left(\mathrm{V}_{\mathrm{H}}-\mathrm{V}_{\mathrm{L}}=10 \mathrm{~V}\right)$ enabled faster accumulation speed. Moreover, for the case of $60 \mathrm{nM}$, the concentration factor reached the reference intensity of $6 \mu \mathrm{M}$ within $6 \mathrm{~min}$. In principle, the preconcentration effect could be enhanced with further increased $\mathrm{V}_{\mathrm{H}}-\mathrm{V}_{\mathrm{L}}$. However, we found Nafion membrane deformation and rapid failure of device after a few minutes when applying voltages $\mathrm{V}_{\mathrm{H}}, \mathrm{V}_{\mathrm{L}}>20 \mathrm{~V}$. The reason could be a low tolerance of the Nafion membrane to Joule heating. It's also noted that the low working voltage of our device holds promise for portable applications.

The fluorescent intensity of the AF-BSA plug for different initial concentrations $(60 \mathrm{pM}, 600 \mathrm{pM}, 6 \mathrm{nM}$ and $60 \mathrm{nM}$ ) at two specific times of $1 \mathrm{~min}$ and $7 \mathrm{~min}$, respectively, was also discussed with the potential difference of $10 \mathrm{~V}$. In the case of $60 \mathrm{pM}$ concentration, the fluorescence intensity at $7 \mathrm{~min}$ reaches that of $60 \mathrm{nM}$ obtained at $1 \mathrm{~min}$, implying an enrichment factor of at least $\sim 10^{3}$. The result indicates that our type of device is able to effectively preconcentrate a low AF-BSA concentration at the pico-molar level. 


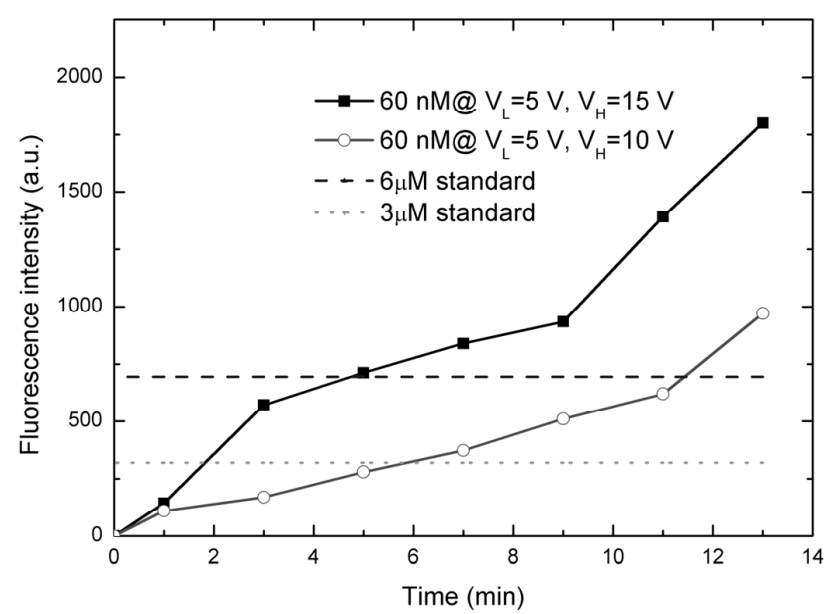

Figure 3. Time-dependent maximum fluorescence intensity of a preconcentrated plug of $A F-B S A(60 \mathrm{nM})$ with two external voltage differences, i.e. $10 \mathrm{~V}\left(V_{L}=5 \mathrm{~V}, V_{H}=15 \mathrm{~V}\right)$ and $5 \mathrm{~V}\left(V_{L}=5\right.$ $\left.\mathrm{V}, V_{H}=10 \mathrm{~V}\right)$, respectively.

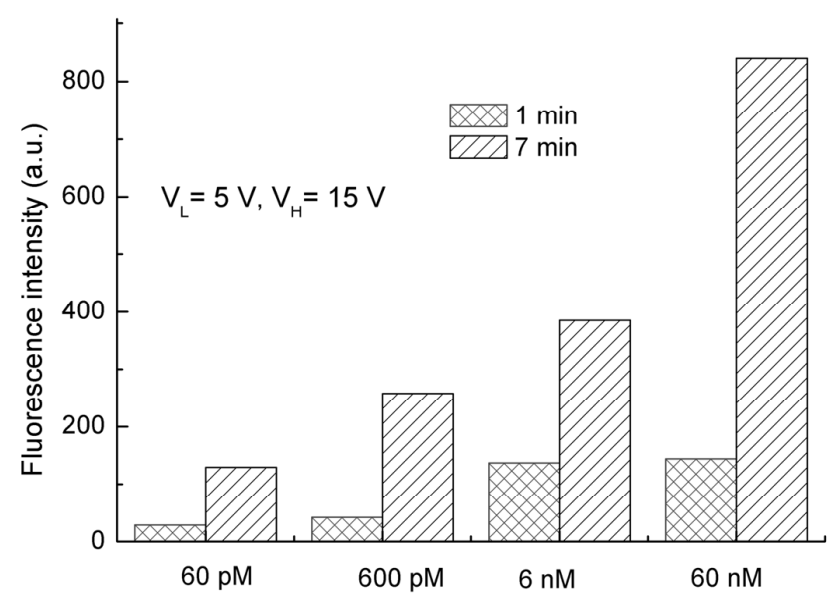

Figure 4. The maximum fluorescence intensity of four $A F-B S A$ concentrations after application of an external voltage $\left(V_{L}=5 \mathrm{~V}\right.$, $V_{H}=15 \mathrm{~V}$ ) for $1 \mathrm{~min}$ and $7 \mathrm{~min}$, respectively.

\section{NUMERICAL SIMULATION \\ Theoretical Background}

In general, the complex interplay of three factors explains the electrokinetic motion of charged molecules in the microfluidic channel, i.e., i) electromigration of the positive and negative ions of the background electrolyte, ii) electrophoresis of the tracer biomolecules, and iii) electroosmosis of the background electrolyte driven by the charge imbalance in the EDL close to a solid-liquid interface [9-10]. In order to study the mechanism of the electrokinetic trapping in our device, a numerical model considering all of the three above factors is proposed.

The model consists of highly coupled equations. The transient Nernst-Planck equation for both types of buffer ions is combined with a proper description of the momentum transfer of the fluid, as expressed by the Navier-Stokes equation, and linked with the electrical potential by applying the Poisson equation. In these three equations, the Nernst-Planck equation is used to describe the transport of positive and negative buffer ions and tracer molecules in the presence of an applied electric field,

$$
\frac{\partial c_{i}(t)}{\partial t}=\nabla\left(D_{i} \nabla c_{i}(t)+z_{i} c_{i}(t) D_{i} \frac{F}{R T} \nabla \phi(t)\right)-\nabla c_{i}(t) \cdot \mathbf{u}(t)
$$

The Poisson equation describes the relation of the electrical potential and the local concentration distribution of buffer ions and charged tracer molecules,

$$
\nabla^{2} \phi=-\frac{\rho_{\mathrm{fix}}}{\varepsilon_{0} \varepsilon_{\mathrm{r}}}-\frac{F}{\varepsilon_{0} \varepsilon_{\mathrm{r}}} \sum_{\mathrm{i}} z_{i} c_{i}(t)
$$

The Navier-Stokes equation and continuity equation are used to describe the motion of an incompressible fluid influenced by a local electrical potential,

$$
\begin{gathered}
\rho \frac{\partial \mathbf{u}(t)}{\partial t}+\rho(\mathbf{u} \cdot \nabla) \mathbf{u}=-\nabla \mathrm{p}+\eta \nabla^{2} \mathbf{u}-F \sum_{\mathrm{i}} z_{i} c_{i}(t) \nabla \phi \\
\nabla \cdot \mathbf{u}=0
\end{gathered}
$$

In these equations, $c_{i}, D_{i}$ and $z_{i}$ are the molar concentration of ionic species $i$, their corresponding diffusion coefficient and corresponding valence, respectively. $F$ is the Faraday constant, $R$ and $T$ represent the molar gas constant and temperature. $\phi$ is the local electric potential, $\rho_{f i x}$ is the volumetric fixed charge density of the membrane, $\varepsilon_{0}, \mathcal{E}_{r}$ are the vacuum permittivity and the relative dielectric constant, respectively, while $\rho$ and $\eta$ are the density and viscosity of the water-based fluid and $\mathbf{u}$ is the fluid velocity vector.

Because of the complexity of the mathematical model, the numerical method is essential to provide a physical explanation of the preconcentration phenomena. Here the software COMSOL Multiphysics ${ }^{\mathrm{TM}}$ (version 3.5a) is used to solve the differential equations for a two-dimensional model with the same geometry as the real device. With an extremely fine mesh (a few nanometers) close to the Nafion membrane, this model can correctly describe the EDL-based effects and the CP-related charge imbalance effects because of the precise solution of the local ion distribution.

\section{Simulation Results}

Figure 5 shows the simulated concentration of positive and negative ions of the electrolyte within the membrane and at the anodic and cathodic compartment side. The curve is obtained along the thin 'neck' in the inserted plot, after applying the external voltage $\left(\mathrm{V}_{\mathrm{H}}=15\right.$ $\mathrm{V}, \mathrm{V}_{\mathrm{L}}=5 \mathrm{~V}, \mathrm{~V}_{\mathrm{B}}=0 \mathrm{~V}$ ) for $7 \mathrm{~min}$. The result shows that the concentration of cations in membrane is higher than that of anions, which is a direct consequence of the perm-selective membrane. Meanwhile, the CP effect is generated with ion-depletion at the anodic side and ion enrichment at the cathodic side. At a small region close to the membrane on the anodic side, there is a local surplus of mobile positive ions (hardly visible from this figure). This indicates the existence of an induced space charge. This effect induces the non-equilibrium EDL, which acts as the origin of the 2nd EOF in the anodic compartment. 


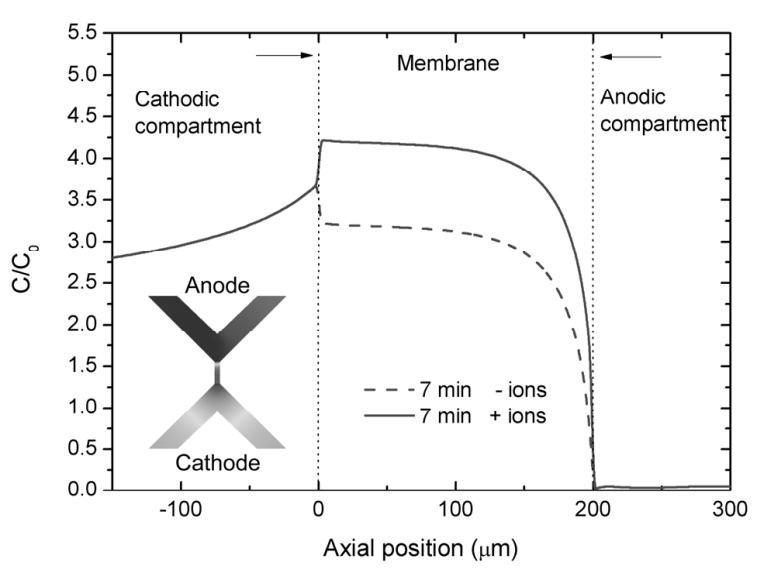

Figure 5. Distribution of positive and negative ions of the electrolyte after application of an external voltage $\left(V_{L}=5 \mathrm{~V}\right.$, $V_{H}=15 \mathrm{~V}$ ) for $7 \mathrm{~min}$. The curves are calculated along the thin 'neck' linking the two compartments (see inset).

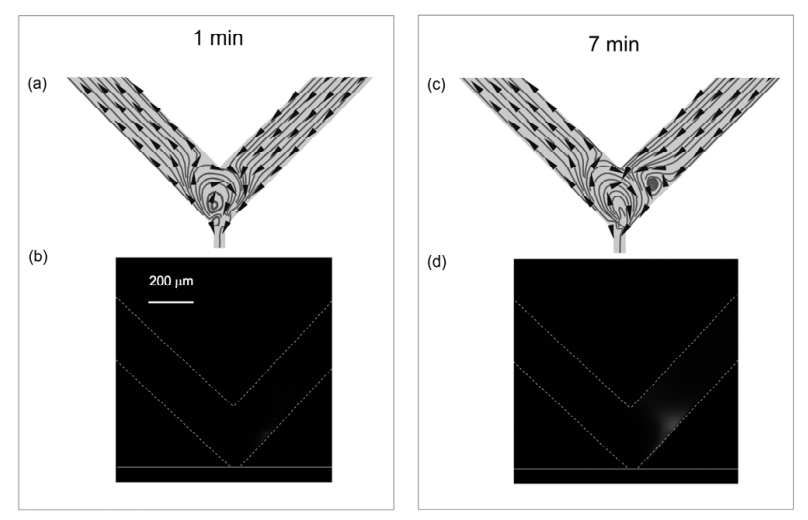

Figure 6. Simulation results for velocity streamlines, as well as experimental fluorescence images after application of an external voltage $\left(V_{L}=5 \mathrm{~V}, V_{H}=15 \mathrm{~V}\right)$ for $1 \mathrm{~min}(a, b)$ and $7 \mathrm{~min}$ $(c, d)$, respectively.

Figure 6 shows the simulated streamlines of fluid in the anodic channel at $1 \mathrm{~min}$ and $7 \mathrm{~min}$, respectively. The flow direction is indicated by the normalized arrows. Because the 2nd EOF is only induced by the non-equilibrium EDL on the membrane side of the sample channel, a vortex-like flow pattern is generated. Based on the co-effect of electrophoresis and EOF of first and second kind, the protein molecules are accumulated at a location near the membrane. The flow pattern indicates that more and more proteins are concentrated near the membrane with increasing time, which well matches the experimental results.

\section{CONCLUSIONS}

We developed a simple protein preconcentrator and numerically studied the working principle of the microdevice. Using low voltages $(\leq 15 \mathrm{~V})$, we have achieved a preconcentration factor of $10^{3}-10^{4}$ for various concentrations of BSA within $10 \mathrm{~min}$, which offers potential for integration into portable microfluidic analytical systems.

\section{ACKNOWLEDGEMENT}

The first two authors contributed equally to this work. The authors are grateful to the Swiss Commission for Technology and Innovation (Project CTI-10004.1 PFNM-NM) and the Swiss National Science Foundation (200020-121558) for funding of this project.

\section{REFERENCES}

[1] M. A. M. Gijs, F. Lacharme, U. Lehmann, "Microfluidic Application of Magnetic Particles for Biological Analysis and Catalysis", Chem. Rev., Vol. 110, pp.1518-1563, 2010.

[2] R. Dhopeshwarkar, R. Crooks, D. Hlushkou, U. Tallarek, "Transient Effects on Microchannel Electrokinetic Filtering with an Ion-Permselective Membrane", Anal. Chem., Vol. 80, pp.1039-1048, 2008.

[3] J.-H. Lee, Y.-A. Song, J. Han, "Multiplexed Proteomic Sample Preconcentration Device Using Surface-Patterned Ion-Selective Membrane", Lab Chip, Vol. 8, pp. 596-601, 2008.

[4] P. Kim, S. J. Kim, J. Han, K. Y. Suh, "Stabilization of Ion Concentration Polarization Using a Heterogeneous Nanoporous Junction", Nano Lett., Vol. 10, pp. 16-23, 2010.

[5] S. J. Kim, L. D. Li, J. Han, "Amplified Electrokinetic Response by Concentration Polarization near Nanofluidic Channel", Langmuir, Vol. 25, pp. 7759-7765, 2009.

[6] S. J. Kim, Y. C. Wang, J. H. Lee, H. Jang, J. Han, "Concentration Polarization and Nonlinear Electrokinetic Flow near a Nanofluidic Channel", Phys. Rev. Lett., Vol. 99, pp. 044501-4, 2007.

[7] M. Shen, H. Yang, V. Sivagnanam, M. A. M. Gijs, "Microfluidic Protein Proconcentrator Using a Microchannel-Integrated Nafion Strip: Experiment and Modeling", Anal. Chem., Vol. 82, pp. 9989-9997, 2010.

[8] J. Q. Guan, Z. F. Dai, C. H. Tung, B. X. Peng, "Fluorescence Enhancement and Photostability of Novel Pentamethine Cyanine in Nafion- $\mathrm{Na}+$ Membranes", J. Fluoresc., Vol. 10, pp.21-26, 2000.

[9] D. Hlushkou, R. Dhopeshwarkar, R. M. Crooks, U. Tallarek, "The Influence of Membrane Ion-Permselectivity on Electrokinetic Concentration Enrichment in Membrane-Based Preconcentration Units”, Lab Chip, Vol. 8, pp.1153-1162, 2008.

[10]A. Höltzel, U. Tallarek, "Ionic Conductance of Nanopores in Microscale Analysis Systems: Where Microfluidics Meets Nanofluidics", J. Sep. Sci., Vol. 30, pp.1398-1419, 2007.

\section{CONTACT}

*H. Yang, tel: 41-21-693 6815; hui.yang@epfl.ch 